\title{
Person marking in Catalan Sign Language (LSC) personal pronouns
}

\author{
Raquel Veiga Busto (UPF, Universitat Pompeu Fabra) \\ raquel.veiga@upf.edu
}

\begin{abstract}
This paper addresses the question of whether the person category is grammaticalized in sign language personal pronouns. Building on Berenz's (1996) Body Coordinates Model, I argue that Catalan Sign Language (LSC) encodes the distinction between first, second and third person. To formalize the analysis, a set of three binary spatial features ([proximal], [central], [mid]) is assumed. The opposition between positive and negative values in this featural system is claimed to be grammatically relevant in the expression of person distinctions, proving that spatial locations are incorporated into the pronominal system, just like they are into other aspects of sign language grammars. The main contribution of this study is that it provides a unified account of person marking that makes it possible to straightforwardly capture person distinctions in the three number values under scrutiny in this investigation (singular, dual and multiple plural).
\end{abstract}

Keywords: person markers, spatial features, Body Coordinates Model, Catalan Sign Language (LSC), personal pronouns

\section{Introduction}

The grammatical category of person encodes the semantic opposition between discourse roles: the speech act participants (i.e. speaker and addressee) and the non-participants in the speech act, also described as 'third party', 'the other category' or 'the non-person' (Benveniste 1971; Cysouw 2001; Siewierska 2004; a.o.). Although much research has been devoted to elucidating whether sign language personal pronouns encode this three-way distinction, no agreement has yet been reached on the matter.

Building on the Body Coordinates Model (Berenz 1996, 2002), this study shows that Catalan Sign Language (LSC) personal pronouns mark first, second and third person differently. Like in Berenz's proposal, the present analysis gets rid of the actual or assigned locations of the referents to account for the marking of person, as the phonological shape of the pronominal form alone proves sufficient to mark person distinctions. I couch the analysis in terms of a featural system which comprises a small set of spatial features ([proximal], [central], [mid]). I further argue that in LSC selecting a feature (which is 'visible' in the phonology by changes in the shape of the sign, i.e. by the use of different person markers) has an impact on the semantics of personal pronouns: reference to the speaker is associated with [+proximal], reference to the addressee with [+central] and reference to a non-participant with either [ø] or [+central, - mid].

The first part of this paper sets up the empirical problem and the motivations for the analysis presented thereafter. Section 2 discusses the notion of person and reports on the 
two main proposals on person marking in the sign language literature. Section 3 offers a brief description of the morphophonological person markers used in LSC and gives arguments for a simplification of the Body Coordinates Model. In the second portion of the paper I develop a new account of person marking in LSC pronouns. Section 4 provides an interim analysis in which the notion of spatial features is implemented. The proposal is further refined in Section 5 by proposing a simpler system that accounts for the relevant articulatory contrasts encoding person values in LSC. Section 6 concludes the paper.

\section{Person distinctions in sign languages}

In LSC, just as in other documented sign languages, singular personal pronouns typically take the form of an index pointing sign. This sign can be directed to the location of a present referent or to a location associated with a non-present referent (Friedman 1975; Lillo-Martin and Klima 1990). The association between discourse referents and spatial locations in sign languages is, then, intimately tied to the act of reference. Crucially, the analysis of the location component of pronominal signs motivates, at least partially, the existence of different proposals regarding the marking of the person category. According to some analyses, the location component of pronominal signs is not linguistic, but gestural (Liddell 1995) and, therefore, pronouns in sign languages fail to encode person values altogether (McBurney 2002). Other studies assume that the category of person is encoded in sign language grammars, but they differ in the number of person distinctions taken to be marked in the system. In the remainder of this section two approaches - the first vs non-first person analysis (Meier 1990; Engberg-Pedersen 1993, 2003; Sandler and Lillo-Martin 2006; Lillo-Martin and Meier 2011; a.o.) and the Body Coordinates Model (Berenz 1996, 2002; Alibašić Ciciliani and Wilbur 2006; Wilbur 2013; Almeida-Silva 2019), which argues in favor of a three-way opposition will be briefly reviewed.

The most widely accepted analysis, first proposed by Meier (1990) for American Sign Language (ASL), argues that the location component of pronouns referring to the addressee and to the non-participant cannot be phonologically specified. This is so because they systematically depend on the actual or the assigned location of the entities they refer to. Following this line of argumentation, it has been proposed (cf. Sandler and Lillo-Martin 2006; LilloMartin and Meier 2011) that these pronouns pick out referents, not classes of referents. This is in sharp contrast to what is observed in first person pronouns, as they are always directed towards the same location, the speaker's chest, and therefore they can be fully phonologically described. This two-person proposal has provided extensive and convincing arguments for the grammaticization of first person pronouns in terms of handshape selection, plural morphology and behavior under role-shift. Although the reasons given work well for ASL and other sign languages, they do not always hold for LSC data.

In her analysis of Brazilian Sign Language (LIBRAS) pronouns, Berenz $(1996,2002)$ takes a different direction when arguing that the actual or the assigned locations of the referents play no part when it comes to person marking. In Berenz's (1996, p. 215) terms: “[p]roduction and perception of the personal pronoun signs are independent of their referential objects". This distinction between phonology and interpretation established in Berenz's work is relevant for the present study, as we aim to provide a linguistic description of person markers, not of reference resolution. Needless to say that the addressee will need to know the context to be able to identify the intended referent of a pronoun, but recognizing a person marker should not require to check the context, provided that the marker in question is part of the pronominal paradigm. An uncontroversial example can be found in first person pronouns. According 
to both Meier (1990) and Berenz (1996), a pointing sign directed toward the signer's chest can be recognized as a first person pronoun, irrespective of whether it refers to the actual signer or to the reported speaker of a role-shift construction. The inevitable question then is: what about second and third person? Leaving aside the entities they refer to (and, by extension, their real or assigned location), is there something in the shape of these signs that constrains the possible person values that might be associated with them? According to Berenz, this is indeed the case. As proposed in her Body Coordinates Model, what is relevant to describe the pronominal form is not the location component, as already mentioned, but rather the 'articulatory array' presented by the signer. This, in turn, is constituted by four coordinates: the handshape, the chest, the eye-gaze and the head. In first and second person, the angle of the coordinates typically approaches zero, meaning that all four coordinates are aligned along the midline of the signer's body, whereas in third person they are not. By using this model, she argues, all three-person values can be formally distinguished.

\section{The category of person in Catalan Sign Language}

\subsection{Morphophonological person markers in LSC}

To investigate whether LSC encodes person distinctions, a sample from two LSC corpora was used. The data was complemented with material elicited by using a combination of acceptability judgments and elicited productions. Elicitation sessions were conducted with two native deaf signers of LSC, a woman and a man, both middle-aged and born and raised in Catalonia.

Prior analyses tend to restrict their scope to singular pronouns only or singular and first person plurals pronouns. This study incorporates other forms of the paradigm into the investigation to determine if it is possible to propose a unified system that accounts for the marking of person irrespective of number values. The sample included singular, dual and multiple plural forms. In LSC, singular forms typically select the index finger handshape (Figure 1); dual pronouns take either the $\mathrm{V}$ or the $\mathrm{K}$ handshape, which moves back and forth between two locations (Figure 2); in multiple plurals the index sign incorporates a (semi) circular movement (Figure 3).

Figure 1: Singular.

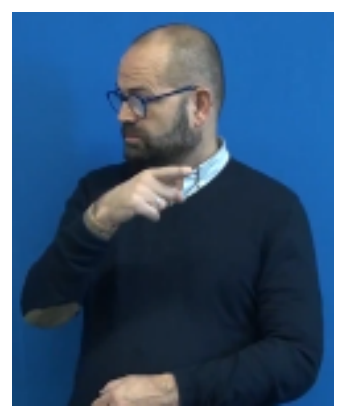

Figure 2: Dual.

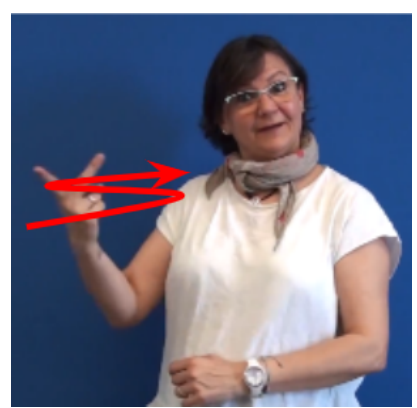

Figure 3: Multiple plural.

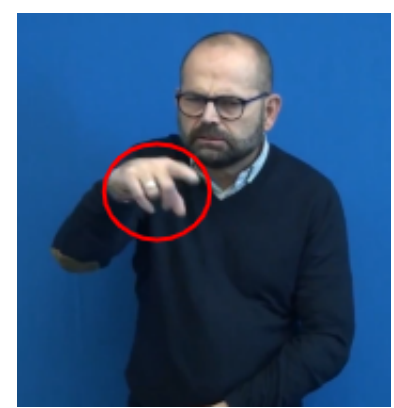

The analysis of the data revealed that in LSC there are systematic differences in the articulation of first, second and third person pronouns across the three number values under investigation. The markers associated with each person value are described below. ${ }^{1}$

\footnotetext{
${ }^{1}$ For a detailed description of person markers in LSC, see Veiga Busto (to appear).
} 
First person is marked by the path movement of the sign, which is directed towards the signer (Figure 2). As a result, there might be contact with the signer's body, particularly in singular forms, as well as a change in the orientation parameter, but neither marker is obligatory.

As in Berenz's model, second person markers involve alignment of handshape, head and gaze. Additionally, second person is signaled by an outward movement, that is, a movement that is not directed towards the signer (Figure 3).

In third person, the handshape and the head are not aligned. This might be accomplished by i) displacing the hand laterally, a strategy extensively used in all three number values (Figure 1); or ii) if the sign is articulated on the same axis established by the head, by directing the pointing downwards (Figure 6) or, much more rarely, upwards. The latter possibility, which is achieved by movement of the wrist or the finger, is only available for singular and plural pronouns. Dual pronouns, which are generally articulated with the palm facing either the signer or upwards, do not allow for the kind of wrist and finger movements responsible for pointing to the lower part of the space in singular and plural forms.

\subsection{The Body Coordinates Model in the light of LSC data}

Although LSC data fits well with the Body Coordinates Model, there are two claims of the model that emerged as problematic during the annotation process. These are the role assigned to the chest and the so-called 'midline avoidance principle' for third person.

In the Body Coordinates Model the chest coordinate is considered to play a central role in distinguishing second from third person. In LSC, however, this claim cannot be taken to hold, as reference to the addressee does not require alignment of the chest (Figure 4). Indeed, alignment of the chest rather appears as an accidental property of second person, considering that in many situations the speech act participants (i.e. speaker and addressee) are facing each other during the conversation. However, this is not a requirement and, in the data used for this investigation, it is far from common. To avoid postulating many exceptions, and as previously proposed to account for Croatian Sign Language (Alibašić Ciciliani and Wilbur 2006) and LIBRAS pronouns (Almeida-Silva 2019), the chest was excluded from the set of relevant coordinates.

According to Berenz's analysis, third person pronouns avoid the midline of the body in order to keep second and third person maximally distinct. As a consequence of this principle, third person pronouns are preferably directed to the ipsilateral side, at least in elicited data. In LSC this rule is not met, as the midline of the signer's body does not impose any constraint on the person values that may be associated with it (see Figure 5 for an example of a third person pronoun directed towards the midline of the signer's body). As a result, it is proposed that it is not the midline, but rather the central space that determines which person values might be associated with it.

The core of the present proposal is that, in terms of person marking, the direction of the head signals the central space. ${ }^{2}$ If handshape and head point in the same direction, then second person is marked. Not surprisingly, the head might be rotated towards one of the sides, but as long as the handshape follows the same direction, second person is marked (Figure 4). Under this view, the midline of the body (and the pointing signs directed to it, as in Figure 5), needs not correspond to the central space.

Third person pronouns, on the other hand, are projected towards the lateral sides with respect to the direction of the head (Figure 1 and 5). Less commonly, third person pronouns

\footnotetext{
${ }^{2}$ Almeida-Silva (2019) provides a similar analysis, but he considers instead the direction of the face.
} 
Figure 4: Second person. Figure 5: Third person. Figure 6: Third person.
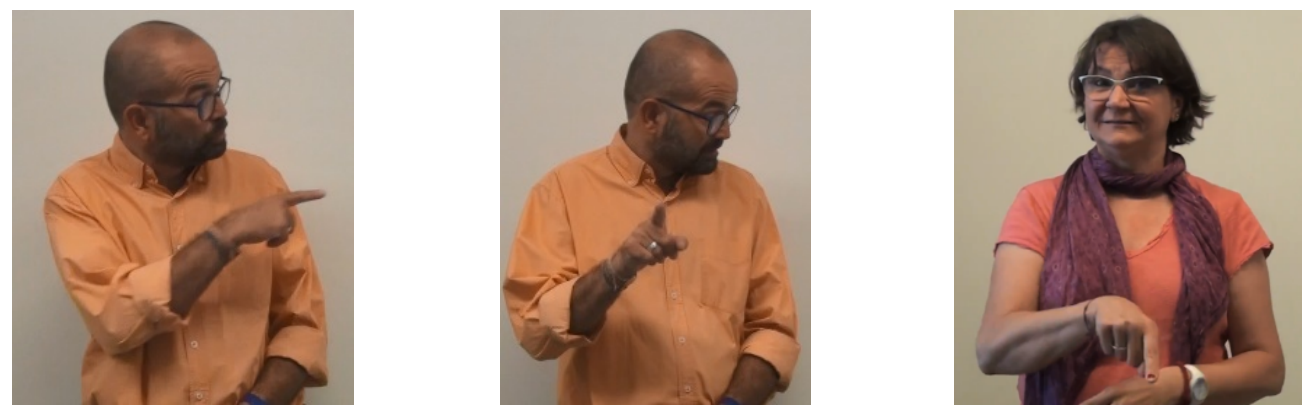

can be articulated in the same direction as the head. As mentioned, in that case misalignment is marked by pointing towards one of the extremes of the vertical, that is, either upwards or downwards (Figure 6). Therefore, there are essentially two different oppositions that appear to be relevant in marking third person: first, one that contrasts central vs lateral locations and second, another one that opposes medial to high and low locations.

\section{Sketching the analysis}

\subsection{Person markers and spatial features}

Based on the aforementioned contrasts, I argue that it is possible to capture LSC person markers by using a unified system composed by a set of three spatial features: [proximal], [central] and [mid]. These features are related to the three spatial planes (as described in Liddell and Johnson 1989; Brentari 1998; a.o.), but they differ slightly in terms of how to identify the values (positive vs. negative) each feature takes.

Unlike other studies, the value of the [proximal] feature is not determined by the angle of the elbow (cf. Liddell and Johnson 1989), but rather by considering whether the path movement of the sign targets the signer's body at some point. This means that the body of the signer can be the only target, as in singular forms; or that the sign can target alternately/ sequentially the signer in addition to some other location, as in dual and plural pronouns. The positive value [+proximal] is hence associated with an inward movement, while outward movements are associated with the negative value [-proximal].

As already mentioned, the value of the [central] feature is not defined by whether the handshape is in line with the chest. Instead, it is resolved by considering whether the handshape aligns with the direction of the head. The negative value [-central] is marked by displacing the handshape to the ipsilateral side or by wrist movement, particularly when pointing to the contralateral side.

Recent studies dealing with the semantics of the signing space consider different oppositions within the frontal plane, such as [up] and [low] (cf. Barberà 2015) or [low], [mid] and [high] (cf. Davidson and Gagne 2014). Such divisions are defined taking into consideration different body parts; for instance, in Barberà (2015), the height of the shoulder and upwards is taken to be the upper part, and below that, the lower area. Although absolute locations appear to play a role in the articulation of personal pronouns (e.g. second person pronouns are usually articulated somewhere between the upper chest and the mouth 
level), in the proposal presented here, the value of [mid] is determined instead by considering whether the handshape is directed parallel or perpendicular to the signer. The negative value [-mid], which in this system includes both downward and upward pointing, is marked by wrist or finger movement, such as flexion or extension. Conversely, whenever the hand is projected perpendicular to the signer, no wrist/finger movement is required and the [mid] feature takes a positive value [+mid]. Such opposition allows us to distinguish forms like the ones in Figure 7 (referring to the addressee) and 8 (referring to a group of non-participants).

Figure 7: Second person [+mid]. $\quad$ Figure 8: Third person [-mid].
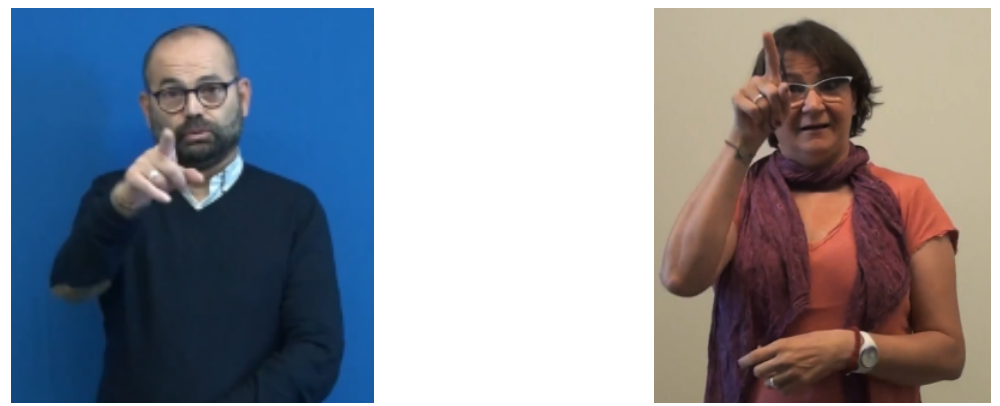

What is pivotal to our proposal of person marking is that the value taken by the features is defined by considering the direction of the handshape in relation to the signer's upper body and head. That is, the value of the features is determined by considering the way the sign is projected into the signing space, the signer himself/herself being the reference point. Figure 9 illustrates the binary distinctions drawn within each feature, where the red arrow corresponds to the positive value and the blue arrow to the negative one.

Figure 9: Spatial features.

(a) [proximal]

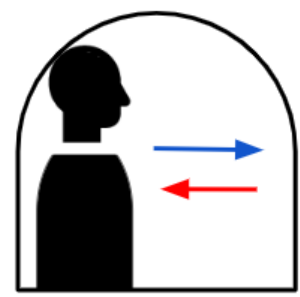

(b) [central]

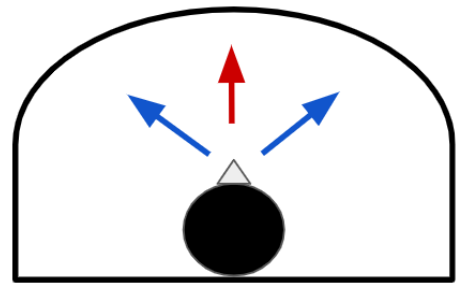

(c) [mid]

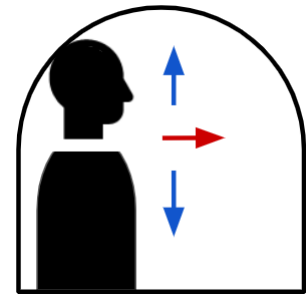

\subsection{Implementing the analysis: singular and multiple plural forms}

The oppositions between positive and negative values for the features in 4.1 prove grammatically relevant in the expression of person distinctions in LSC. Indeed, for singular and multiple plural forms, the matrices in Table 1 allow us to characterize the articulatory contrasts for the marking of person described in section 3.1.

First singular and plural forms (Figures 10a, 10b and 10c) all share the [+proximal] property, as the pronoun targets at some point the body of the signer. The rest of the features vary: only the singular and the inclusive plural (meaning 'I and you pl.' or 'I, you and some other(s)') are [+central], while the exclusive form ('I and others, not you'), being laterally displaced, takes the negative value [-central]. The pronoun is projected perpendicularly to the signer only in the singular, hence the [mid] feature takes a negative value in multiple plurals. 
Table 1: Feature matrices for singular and multiple plural pronouns.

\begin{tabular}{|c|c|c|}
\hline Number value & Person value / reference set & Features \\
\hline \multirow[t]{3}{*}{ Singular } & 1 & {$[+$ prox,+ cent, + mid $]$} \\
\hline & 2 & {$[-$ prox,+ cent,+ mid $]$} \\
\hline & 3 & {$[-$ prox,- cent, \pm mid $][-$ prox,+ cent,- mid $]$} \\
\hline \multirow[t]{4}{*}{ Multiple plural } & $1+2+2 ; 1+2+3$ & [+prox, +cent,-mid] \\
\hline & $1+3+3$ & [+prox,-cent, -mid] \\
\hline & $2+2+2 ; 2+3+3$ & {$[-$ prox,+ cent,+ mid $]$} \\
\hline & $3+3+3$ & {$[-$ prox,- cent, \pm mid $][-$ prox,+ cent,- mid $]$} \\
\hline
\end{tabular}

Figure 10: First person.

(a) Singular

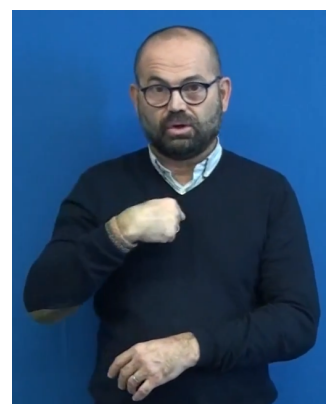

(b) Inclusive plural

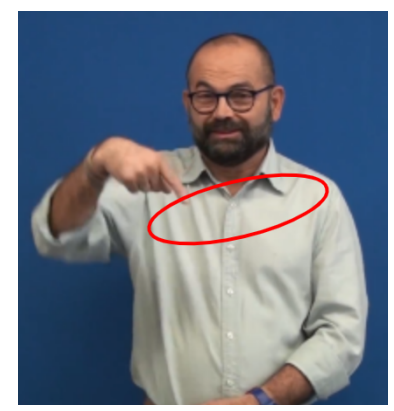

(c) Exclusive plural

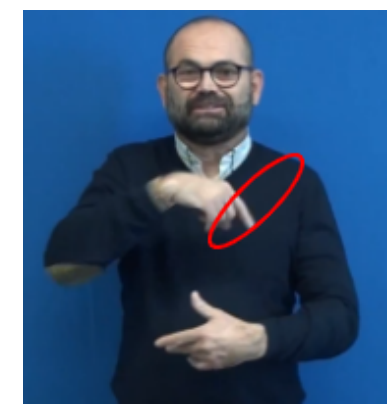

Unlike first person, second person pronouns (Figures 7 and 3), irrespective of the number value, all take the same features, namely [-proximal, +central, +mid]. Additionally, second person pronouns show no articulatory distinction depending on whether the plural pronoun refers to multiple addressees $(2+2+2)$ or to a set containing the addressee(s) and one or multiple non-participants $(2+2+3,2+3+3 \ldots)$. The forms are [-proximal], as the path movement goes outwards; on the horizontal plane they are aligned with the direction of the head, so they are [+central]; and with respect to the vertical axis, they are directed to the signer's body perpendicularly, so they take the $[+$ mid] feature.

Figure 11: Third person.

(a) Singular

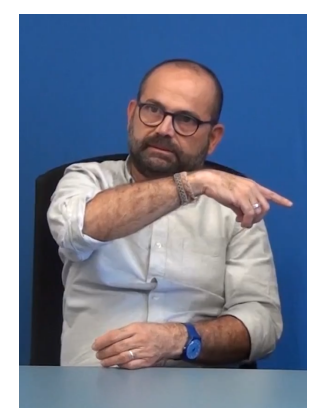

(b) Multiple plural

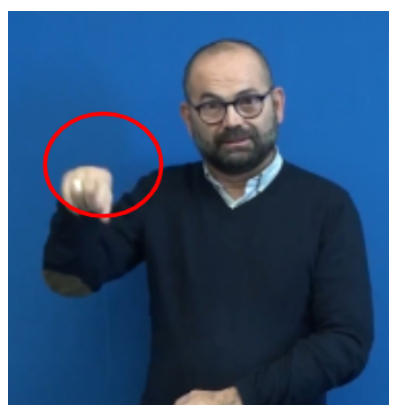

(c) Singular

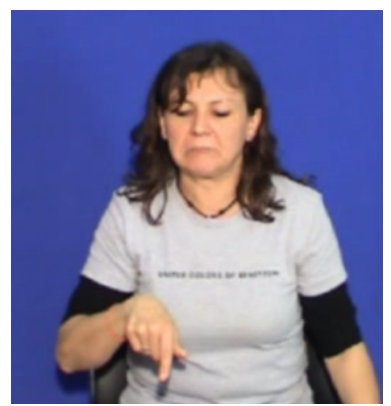

(d) Multiple plural

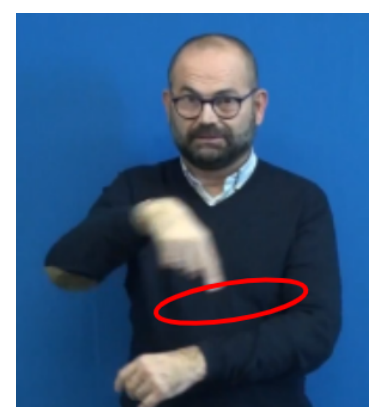

For third person there are two options, which represent the two ways in which a third person 
pronoun might be misaligned. Again, the number value does not change the sequence of features a third person pronoun takes. In the first constellation of features in Table 1, which represents the most common articulation of third person (Figures 11a and 11b), the pronoun is [-proximal], as it does not target the signer; since it is directed to the lateral sides of the horizontal plane, it is [-central], and both [+mid] and [-mid] values are possible.

The second option in the table stands for attested, but less frequent third person forms (Figures 11c and 11d). The forms in question are [+central], that is, aligned with the head, and [-mid], as the pointing is projected either downwards or upwards in the vertical axis. Either way, in both cases third person pronouns are directed to peripheral locations: they are pushed either to the edges of the horizontal plane (i.e. to the lateral sides), or to the upper and lower extremes of the vertical one (i.e. parallel to the signer's body).

\subsection{Dual pronouns and implications for the analysis}

Unlike what is observed in singular and multiple plurals, some person oppositions may go missing in dual forms. This is not typologically surprising, as it is not uncommon for the dual value to encode less distinctions than singular and plurals do. Besides, dual pronouns do not point downwards, so it is not possible to draw a distinction with the [mid] feature, as there is no opposition at play. Therefore, the constellations of features for dual pronouns would look like in Table 2.

Table 2: Feature matrices for dual pronouns.

\begin{tabular}{lll} 
Number value & Reference set & Features \\
\hline Dual & $1+2$ & [+prox, + cent $]$ \\
& $1+3$ & [+prox, - cent $][+$ prox,+ cent $]$ \\
& $2+2 ; 2+3$ & {$[-$ prox,+ cent $]$} \\
$3+3$ & {$[-$ prox,- cent $][-$ prox,+ cent $]$}
\end{tabular}

Since [mid] does not play any role in the marking of person values in dual forms, when a dual pronoun takes the [+central] feature, some contrasts cannot be drawn. Their interpretation is typically made clear in actual discourse, but when taken out of context, some forms become undistinguishable. For instance, if a dual referring to two non-participants is articulated in a [+central] location (Figure 12) it cannot be formally differentiated from a second person dual (Figure 13).

Figure 12: Dual (3+3).

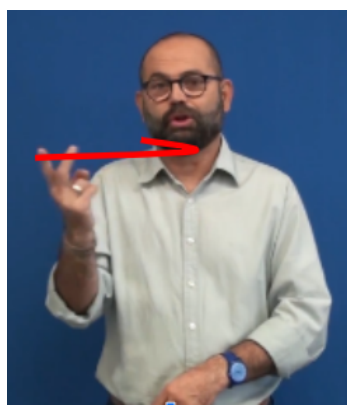

Figure 13: Dual (2+2).

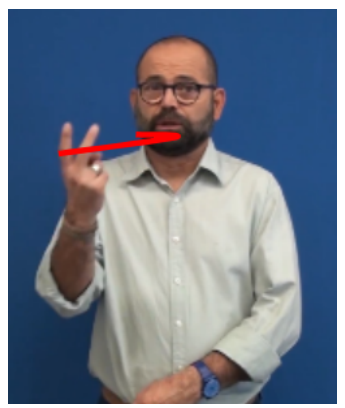

Figure 14: Dual (3+3).

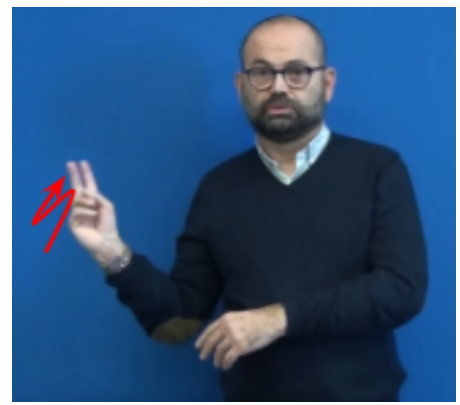


This reveals that the features [-proximal, +central] do not force a second person reading, as they are compatible with both second and third person duals. In other words, every second person dual is associated with the features [-proximal, +central], but not every dual that takes these features is necessarily a second person dual. The same observation applies to first person inclusive and exclusive duals when the exclusive pronoun is articulated in a central location. Importantly, despite the fact that dual pronouns appear to be counterexamples to the analysis proposed so far, it is not suggested that duals cannot mark person oppositions, but rather that they are more likely to neutralize distinctions otherwise widespread and systematic in the grammar. This is confirmed by the fact that whenever a dual pronoun is articulated in a non-central location (Figure 2 and 14), the distinction between second and third person as well as the distinction between inclusive and exclusive first person are present in the system.

\section{Proposal: association of features with semantic values}

The case of dual pronouns presented in the previous section evidences the fact that some features might be unspecified. In other cases, certain specifications might seem trivial or redundant (such as [central] for first person singular, since there are no forms that contrast only by changing the value of that feature). Hence, in order to obtain a more efficient system, we need to isolate the basic distinctive features of each person value (Table 3).

For the first person singular, the only relevant feature we need to specify is [+proximal]. This feature is observable in the articulation of first person pronouns by the path movement of the sign, which moves towards the signer. The [+proximal] feature is also true for any set containing the referential element speaker (i.e. first person dual and multiple plurals), but in these cases we might want to detail the features further if we were to capture the distinction between the inclusive and the exclusive interpretation, as the forms contrast by changing the value of the [central] feature. In fact, since inclusive and exclusive first person dual and multiple plural pronouns involve reference to at least two distinct person values, it comes as no surprise that we would need at least two features to account for their marking. As it could be predicted, sets containing the referential elements speaker and addressee take the [+proximal] and [+central] features (i.e. the features corresponding to first and second person). On the other hand, sets containing the speaker and a non-participant take the [+proximal] and [-central] features (i.e. the features associated with first and third person). Again, if the exclusive pronoun selects a [+central] articulation, the distinction with the dual inclusive would be neutralized.

Second person, in all three number values, is consistently defined by taking the [+central] feature, and no further distinctions are needed. The selection of the [+central] feature is made visible by aligning the direction of the handshape, head and eye-gaze. The fact that the [proximal] feature is not active implies that the sign is articulated with an outward movement.

Finally, third person pronouns present us with two alternatives. The first one ([ø]), which corresponds to its most common articulation, reveals the fact that third person pronouns are not only negatively defined in their semantics (as being the 'non-person', i.e. whoever or whatever is not the speaker nor the addressee), but also in their articulation (as being what is neither proximal nor central, i.e. by the absence of the features of first and second person). In articulatory terms, this means that the sign takes an outward movement and that is laterally misaligned, meaning that the direction of the head and the hand does not coincide. Besides, when the pronoun takes the [-central] articulation, the value of the [mid] feature does not 
need to be specified, as the pronoun can take any direction on the vertical axis without impacting its interpretation. Even if additional semantic interpretations or constraints on the direction a pronoun might take could be posited (cf. Barberà 2015), the assignment of the person value (reference to the non-participant(s), i.e. third person) would remain constant. The second sequence of features for third person stands for [+central] forms that are misaligned by being projected towards the extremes of the vertical axis (i.e. [-mid]). As this is not possible for duals, when [+central], a third person dual would take the same features as the second person.

Table 3: Association of features and person values

\begin{tabular}{lll}
\hline 1 & {$[+$ prox $]$} & Singular \\
2 & {$[+$ cent $]$} & Singular, dual, plural *dual $(\mathbf{3 + 3})^{*}$ \\
3 & {$[\emptyset]$} & Singular, dual, plural \\
& {$[+$ cent, - mid $]$} & Singular, plural \\
$1+2 ; 1+2+2 / 3$ & {$[+$ prox, + cent $]$} & Dual, plural *dual $(\mathbf{1 + 3})^{*}$ \\
$1+3 ; 1+3+3$ & {$[+$ prox, - cent $]$} & Dual, plural \\
\hline
\end{tabular}

\section{Summary}

In LSC, accounting for the different marking of first, second and third person is possible by using a modified version of Berenz's (1996) Body Coordinates Model that i) reduces the set of coordinates, ii) rules out the existence of the 'midline avoidance' principle for third person and iii) simplifies the system by providing a set of features ([proximal], [central], [mid]) that stand for the way pronouns are projected into the signing space. Under this view, the problem of incorporating the location component of second and third person in the phonological description simply disappears, as the value of every feature is invariably resolved by taking in consideration the signer's body, not the actual or the assigned location of the referents.

According to this proposal, first and second person pronouns can be described by using only one feature and they have constant and predictable forms. More variation is found in third person pronouns, as they do not always show the same sequence of features. As a result, i) the form of a third person pronoun is recognizable and can be described, but it cannot be predicted; and ii) when third person is central, the distinction between second and third person might be neutralized, as in the case of dual pronouns.

All in all, this analysis suggests that spatial locations are incorporated into the LSC pronominal system and that selecting a feature has an impact on the interpretation of pronominal signs. More precisely, in LSC different regions within the signing space are associated with reference to different discourse roles: [+proximal] locations presuppose reference to the speaker, [+central] locations are associated with reference to the addressee and otherwise ([ø] or [+central, -mid]) they are interpreted as denoting a non-participant in the speech act.

\section{Acknowledgments}

I would like to thank Josep Quer, as well as my informants, Santiago Frigola and Delfina Aliaga. I am also grateful to the audience of FEAST 2020 and the members of the Research 
in Sign Languages and Syntax and Semantics seminars at UT Austin for their valuable feedback on previous versions of this presentation. This research has been supported by the Spanish Ministry of Economy and Competitiveness (MINECO and FEDER, ClauseCombi2 FFI2012-36238 and GramRefLSC FFI2015-68594-P, BES2013062848), the Government of the Generalitat de Catalunya (2017 SGR 1478) and the European Union (Horizon 2020 SIGNHUB 693349).

\section{References}

Alibašić Ciciliani, Tamara, and Ronnie B. Wilbur. 2006. "Pronominal system in Croatian Sign Language”. Sign Language and Linguistics 9 (1/2):95-132. doi:10.1075/sl1 .9 . 1.07ali.

Almeida-Silva, Anderson. 2019. "A (in)definitude no sintagma nominal en Libras: Uma investigação na interface sintaxe-semântica”. PhD thesis, Universidade Estadual de Campinas.

Barberà, Gemma. 2015. The Meaning of Space in Sign Language. Reference, Specificity and Structure in Catalan Sign Language Discourse. Boston: Mouton de Gruyter / Ishara Press.

Benveniste, Emile. 1971. Problems in General Linguistics. Coral Gables, FL: University of Miami Press.

Berenz, Norine. 2002. "Insights into person deixis". Sign Language and Linguistics 5 (2): 203227. doi:10.1075/sl1.5.2.06ber.

— . 1996. "Person and Deixis in Brazilian Sign Language". PhD thesis, University of California, Berkeley.

Brentari, Diane. 1998. A prosodic Model of Sign Language Phonology. Cambridge, MA: MIT Press.

Cysouw, Michael. 2001. “The Paradigmatic Structure of Person Marking”. PhD thesis, Radboud University Nijmegen.

Davidson, Kathryn, and Deanna Gagne. 2014. "Vertical representations of quantifier domains". In Proceedings of Sinn und Bedeutung 18, ed. by Urtzi Etxeberria et al., 110-127.

Engberg-Pedersen, Elisabeth. 2003. "From pointing to reference and predication: pointing signs, eyegaze, and head and body orientation in Danish Sign Language". In Pointing. Where Language, Culture and Cognition Meet, ed. by Sotaro Kita, 269-292. Mahwah, NJ: Lawrence Erlbaum.

- . 1993. Space in Danish Sign Language. The Semantics and Morphosyntax of the Use of Space in a Visual Language. Hamburg: Signum-Verlag.

Friedman, Lynn. 1975. “Space, time and person reference in American Sign Language”. Language 51 (4): 940-961. doi:10. 2307/412702.

Liddell, Scott K. 1995. "Real, surrogate, and token space: grammatical consequences in ASL”. In Language, Gesture and Space, ed. by Karen Emmorey and Judy Reilly, 19-41. Hillsdale, NJ: Lawrence Erlbaum.

Liddell, Scott K., and Robert E. Johnson. 1989. "American Sign Language: the phonological base”. Sign Language Studies 64 (1): 195-277. doi:10.1353/sls . 1989.0027.

Lillo-Martin, Diane, and Edward S. Klima. 1990. "Pointing out differences: ASL pronouns in syntactic theory”. In Theoretical Issues in Sign Language Research 1, ed. by Susan D. Fisher and Patricia Siple, 191-210. Chicago: The University of Chicago Press. 
Lillo-Martin, Diane, and Richard P. Meier. 2011. 'On the linguistic status of 'agreement' in sign languages”. Theoretical Linguistics 37 (3/4): 95-141. doi:10.1515/thli .2011.009.

McBurney, Susan. 2002. "Pronominal reference in signed and spoken language: Are grammatical categories modality-dependent?” In Modality and Structure in Signed and Spoken Languages, ed. by Richard P. Meier, Kearsy Cormier, and David Quinto-Pozos, 329369. Cambridge: Cambridge University Press.

Meier, Richard P. 1990. "Person deixis in American Sign Language". In Theoretical Issues in Sign Language Research 1, ed. by Susan D. Fisher and Patricia Siple, 175-191. Chicago: The University of Chicago Press.

Sandler, Wendy, and Diane Lillo-Martin. 2006. Sign Language and Linguistic Universals. Cambridge: Cambridge University Press.

Siewierska, Anna. 2004. Person. Cambridge: Cambridge University Press.

Veiga Busto, Raquel. to appear. “The category of person in Catalan Sign Language (LSC) personal pronouns”. Revista d'Estudis Catalans/ Zeitschrift für Katalanistik.

Wilbur, Ronnie B. 2013. “The point of agreement: Changing how we think about sign language, gesture, and agreement”. Sign Language and Linguistics 16 (2): 221-258. doi:10 . 1075/sll.16.2.05wil. 